\title{
Problems Facing Promotion of Astronomy in Arab Countries
}

\author{
Anas M. I. Osman \\ National Research Institute of Astronomy and Geophysics, Helwan, \\ Egypt. e-mail: amiosman46@hotmail.com
}

\begin{abstract}
Promotion of astronomy in Arab countries is facing many scientific and technical problems. Teaching astronomy starts very late in schools, with very simple and limited courses. Many teachers lack a suitable astronomical background, which can lead to incorrect understanding by students of many astronomical ideas and phenomena. Teaching astronomy at higher levels is also very limited, for example: aomng the 16 universities in Egypt, astronomy is taught in only two faculties of science, just for two years. Graduate students find many difficulties in obtaining jobs related to astronomical activities and this is a serious limitation on the attraction of the study of astronomy. On the other hand, astronomical institutions are suffering from a serious lack of the new sophisticated equipment, while the budget allotted for maintenance is very small, and there is a serious shortage of technical staff. The training of astronomers and technicians is badly needed, since good research work depends on modern technological equipment and the complicated software packages used in controlling such equipment and in data analysis. Good libraries are needed for promotion of astronomy especially, the Internet facilities available for the staff is very limited. The effects of culture are very clear; many authorities in developing countries believe that astronomy is a luxury. Finally, most of astronomers are engaged with a lot of administration for all matters, so the free time left for science is very limited.
\end{abstract}

\section{Introduction}

\subsection{Ancient astronomy}

Astronomy in Arab countries is an old and important science, and has flourished throughout history. This is very clear from the time of the ancient Egyptians, who observed the motions of the Sun, Moon and planets and the heliacal rising of stars, especially Sirius, whuch were used to establish the solar and sidereal calendars. The Egyptian calendar begins with the heliacal rising of Sirius, which defined the beginning of the Nile's annual flooding. They used astronomical means to construct and orient pyramids and temples as well. The sun shines on February 22nd October 22nd, every year, on the statue of Ramses II. One of these days was his birthday and the other was the anniversary of his coronation. Later, the Alexandria school was founded in the city which was the capital of the country during the time of Ptolemies, and much was achieved by many 
scientists and astronomers. Among them were Aristyllus, who was interested in stellar catalogues, and Aristarchus, who measured the relative distance of the Sun and the Moon and estimated the real diameters of these objects and the size of the Earth relative to the Sun and Moon. Eratosthenes determined the Earth's radius using the inclination of the Sun at noon in two different cities, Alexandria and Syene near Aswan, knowing the distance between them. Hipparchus was a great astronomer; he observed the stars positions and completed a star catalogue and discovered the phenomenon of precession. He devised a sophisticated geocentric model of the universe capable of describing and predicting most observed motions of the celestial bodies in the sky. The Julian calendar had been introduced in 46 B.C., by Sosigenes at the time of Julius Caesar. In the second century A.D. Ptolemy wrote his famous book, Almagest, in which he summarized all the then-current knowledge of astronomy in thirteen volumes. He extended the star catalogue to 1022 entries, correcting older reported positions for precession. The best-known contribution of Ptolemy in his book was a new cosmological model for the planetary system in which he assumed that the Earth is near the center of the system. The method for predicting the positions of the Sun, Moon and planets was called the epicycle theory.

On the other hand, the ancient Babylonian civilization in Iraq played an important rule in the field of astronomy. As early as 3000 B.C., they had developed a solar calendar using the Sun and the Moon to keep time and mark the seasons. They observed the motions of the Sun, Moon and the five visible planets among the stars. They recorded these motions and discovered and recorded the annual apparent path of the Sun through the sky, which is called the ecliptic. Also, they carefully studied the Moon and its phases and found that, there are roughly twelve complete changes of the Moon in a year. By 600 B.C., they were able to predict the future positions of the moon and planets within the band of constellations, which they called the Zodiac.

\subsection{Arab astronomy}

The Arab contribution to astronomy was not less important than that of other civilizations. In 640 A.D., much of the Alexandrian knowledge was transferred to Arabs, who further developed mathematics and astronomy. They had compiled tables for determining the positions of planets, the first visibility of the lunar crescent, times of prayer for different geographic longitudes andfinding the direction of Mecca, and they constructed curves for astrolabes and quadrants. As an example of Arab advancement, we note the recorded measurement of the Earth's circumference made near Baghdad in the year 820. The result was only $4 \%$ too large. Similarly, the Arab astronomer, Al-Battani had only $4 \%$ error in his measurement of the eccentricity of the Earth's orbit. He wrote his famous book Elzig, that contains astronomical tables for the motion of the Sun after long-term observations. These tables were used widely in Europe after they had been translated into Latin. From his observations, Al-Battani could define the time at which the length of the day equalled that of the night, and the length the year, with very high accuracy. Accurate observations helped him to obtain new important information regarding the orbits of Earth, Sun and Moon. The work of Ibn Yunis and his Hakimit Tables were very important in the field of astronomy. He observed a solar eclipse from an observatory on Mokattum 
mountain near Cairo and calculated the period of the Earth's rotation from the time and height of the solar disk before and after the eclipse. Al Byruni, Al Faraghani, Ibn El Heitham and others were also important Arab astronomers. Around 1000 A.D., the Islamic Empire had spread to Spain and astronomical tables were published with the reference longitude in Córdoba. Many astronomical phenomena (sunrise, sunset, moonrise, moonset, twilight, lunation...etc.) are strongly related to Moslem traditions (prayer time, Ramadan Fasting, beginning of lunar months and Pilgrimage). In spite of this fact, and in spite of their previous achievments, Arab countries did not continue their success in the field of astronomy. Nowadays, promotion of astronomy in Arab countries is facing many scientific and technical problems, the solutions of which need all efforts, if we are to push astronomy forward in this present era of space exploration.

\section{Problems Facing Promotion of Astronomy in Arab Countries}

\subsection{Astronomy education}

Education in Astronomy in Arab countries is still limited despite its importance for public and Moslem traditions. On the level of elementary schools and popular education, the astronomical knowledge and information included in books and presented and explained by teachers is very limited. Many teachers do not have a suitable astronomical background to explain the different astronomical phenomena, since they graduated in different branches of science. This serious factor affects directly the accuracy of astronomical information transmitted to the students, and is the main reason for some incorrect and inaccurate understanding and explanations. On the level of preparatory and secondary schools, astronomy is taught as small parts of geography and physics, concentrated on the solar system and the different elements of each planet, beside short notes about stars, galaxies and the seasons. The educational aids and demonstration facilities are very limited, for example, planetaria are not available in most cities in Arab countries. On these levels, training courses for teachers, through official seminars and visits to observatories and open discussions with astronomers, are badly needed. These courses must be sponsored directly by ministries of education. Also, the education authorities in Arab countries are requested to support the introduction of astronomy into secondary schools, to attract amateur astronomers and interested students to study astronomy in universities and higher schools. At the level of universities or higher schools, a subsidiary elementary course is taught in some geography and surveying departments, while some Arab universities teach a general course in astronomy for the first-level students, but not as a compulsory course. On the other hand, the lack of astronomy departments in Arab universities is very clear. Physics departments offering astronomy and astrophysics courses for the B.Sc. degree in physics and astronomy are very few, in comparison to the large number of universities. For example, among 16 universities in Egypt including 21 faculties of science, astronomy is taught only in two departments. The Astronomy Department of Cairo University, Faculty of Science awards the B.Sc. and the higher degrees of M.Sc. and $\mathrm{Ph} . \mathrm{D}$. in astronomy, although basic astronomical and astrophysical courses are taught in the third and forth years of study. This restricts the number of the students, who want to study astronomy, since they have no background in 
that science as they do in other sciences which are taught in the first years. The second department in Egypt is the Department of Astronomy and Meteorology in Al Azhar University, where only B.Sc. in astronomy or meteorology can be awarded. This causes a serious shortage of graduate astronomers who can work in observatories or astronomical institutions. As an average, there are two or three graduate students per year, and some years none graduate. Due to the absence of graduate astronomers, the astronomical institutions assign jobs for graduates from physics or mathematics. Also, in Jeddah and King Saud Universities in Saudi Arabia, there are two departments, although there are no observatories in which to work. In Jordan, there is an Institute for Astronomy and Space Science which awards the M.Sc. degree, although there are no astronomy departments in Jordan's universities offering undergraduate studies in astronomy. Accordingly, the chances to pick a good astronomer to join in high-standard astronomical activities are limited. The limitations of astronomy education in Arab countries are the main reason for the serious shortage in the number of high-standard graduate astronomers to teach at different levels and to do good research work.

\subsection{Astronomical culture}

In spite of the relation between religion and astronomy in Arab countries, where many astronomical phenomena have been used in daily life (Prayer times, etc.), much of the public does not feel the relation and its benefits. Many people know nothing about stars and planets, or even the Sun and Moon, except their positions in the sky. Moreover, some educated persons do not know the difference between astronomy and meteorology or the difference between astronomy and astrology. This confusion is mainly due tothe lack of astronomical background. The simplified popular astronomy books are very rare and are often written by non-astronomers, while publishers are not willing to publish astronomy books, or even translated ones, because of the problem of distribution. Although some planetaria are found nowadays in some big cities, their numbers are still not enough to play effective role in popularizing astronomy among the public and youth. Astronomical societies in Arab countries are very weak and very small in number. In most cases, they depend upon the membership of some amateurs and students, who cannot prepare or organize a public seminars or general lectures in the field of astronomy. Accordingly, contact between astronomers and the public is absent and astronomical information is not transferred to society. Finally, TV and broadcasting can play a serious role in enhancing the astronomical background of the public. They can present programs and films about the heavens and space exploration and explanations of some astronomical phenomena. Unfortunately, there is no interest in this type of film or program and people who can prepare and present these programs are very rare and face many obstacles in these jobs.

\subsection{Limited jobs for graduate astronomers}

Although the number of graduate astronomy students is very small, there are not enough jobs for them. The limited number of observatories and astronomical institutions in Arab countries decreases the chance for graduate students to find jobs. Each year, observatories and astronomy departments have about two 
jobs available for brilliant graduates. Because of the economical situation, the students prefer to study other branches of science rather than astronomy, to have the chance for work after graduation. This is one of the main factors restricting the spread and promotion of astronomy in Arab countries.

\subsection{Training}

Training is very important for astronomers and technical staff if they are to use and to run the new sophisticated instruments used nowadays in astronomy. The use of CCD cameras and other modern detectors for astronomical observations requires a high standard from astronomers and technicians. In addition, training on software packages is badly needed, especially those packages used in controlling the various detectors and in analyzing the observational data. Good research work cannot be done without modern equipment and good computing facilities. Although, Egypt for example, has a 2-m telescope and many Arab countries could buy other telescopes very easily, there is no guarantee that they would be run continuously and produce good research work. The budget allotted for maintenance is very small compared to the price of the instruments themselves, so a telescope may stop working at any time. This problem can be solved by inviting experts from outside and sending students and technicians to other observatories and institutions in developed countries, where they can receive a real training.

\subsection{Libraries, books and periodicals}

There is a serious shortage in astronomy books and periodicals in most Arab libraries, since most of them are published outside the Arab region and are quite expensive. Since reference works serve only a very limited number of astronomers and students, the budget allotted by the authorities is very small, correlated to these numbers in spite of the high prices. Foreign astronomy books translated into Arabic are very rare, while our libraries do not contain Arabic astronomical references. Because of this situation, new researches and discoveries in the field of astronomy are not available to Arab astronomers and students. The Internet service needs to be introduced to all Arab astronomical institutions if all these difficulties are to be overcome.

\subsection{Geographical position and international contacts}

Because of the geographical position of most Arab countries, they are isolated from the advanced Western countries with their famous observatories and institutions. It is difficult for Arabian astronomers or students to attend a meeting or conference in Europe, since this needs a lot of money for travel, while that is no problem for any European, who can travel through Europe by train and without entry visas. This situation prevents many useful contacts with the advanced institutions in the fields of training and joint research work on which the promotion of astronomy in Arab countries can take place. 


\section{Suggestions}

In order to overcome the many difficulties facing the promotion of astronomy in Arab countries we recommend:

- Introducing astronomy as a special course for students in secondary schools.

- Training of teachers at all levels of education in the field of astronomy.

- More astronomy departments must be founded for more astronomers.

- Constructing observatories in Arab countries to attract graduate students and amateurs.

- Training of young astronomers and technicians abroad to improve their standard.

- Delivering popular lectures and seminars for public.

- It is important to allot a good budget by authorities for different astronomical activities.

- Introducing astronomy as a general compulsory course for the students of the first level in the universities.

- Encouraging the translation of astronomy books into Arabic.

- Ordering books and periodicals in the field of astronomy and increasing the budget for libraries.

- Supporting the publishing of simplified books for the public at cheap prices.

- Intoducing Internet service into all Arabic institutions. 